\title{
Galenus-von-Pergamon-Preis 2015
}

\section{Ausgezeichnete Innovatoren}

\author{
Zum 30. Mal wurde der Galenus-von-Pergamon- \\ Preis verliehen, und zwar in den vier Kategorien \\ Grundlagenforschung, Primary Care, Specialist Care \\ und Orphan Drugs. Außerdem wurde in Berlin mit \\ dem CharityAward ehrenamtliches Engagement \\ in der Gesundheitsversorgung ausgezeichnet.
}

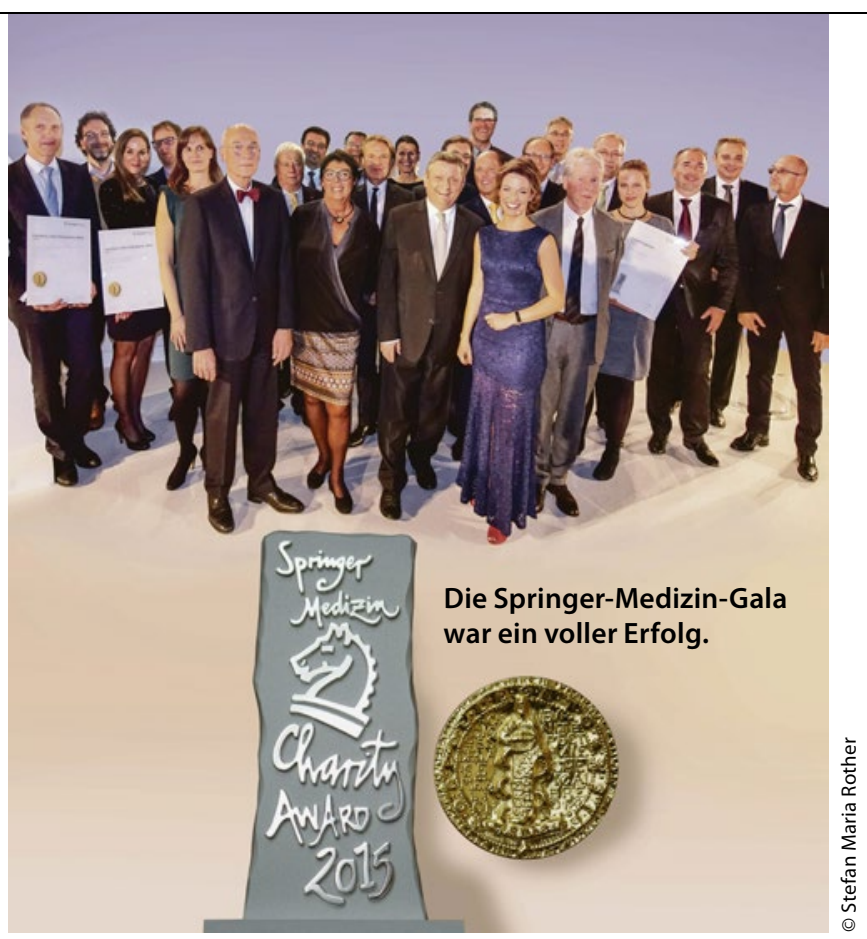

$\mathrm{D}$ ie Preisträger des Galenus-vonPergamon-Preises und des CharityAwards 2015 stehen fest: Am Abend des 15. Oktober haben der Bundesminister für Gesundheit Hermann Gröhe und der Vorsitzende der GalenusJury Prof. Erland Erdmann zusammen mit der Vizepräsidentin Prof. Marion Kiechle die von Springer Medizin gestifteten Preise in Berlin verliehen.

Zur Springer Medizin Gala kamen über 200 geladene Gäste im Axica am Brandenburger Tor zusammen, um hautnah mitzuerleben, welche herausragenden Leistungen in der Arzneimittelforschung und in ehrenamtlichem Engagement ausgezeichnet werden. Beides, pharmazeutischer Fortschritt und Ehrenamt, sind unverzichtbare Leistungen, die $\mathrm{zu}$ einer besseren Gesellschaft beitragen.

\section{Preisträger in vier Kategorien}

Auch in diesem Jahr wurden GalenusPreise in vier Kategorien vergeben: pharmakologische Grundlagenforschung, Primary Care, Specialist Care und Orphan Drug.

Den Galenus-von-Pergamon-Preis in der Kategorie beste pharmakologische Grundlagenforschung nahm in diesem Jahr Professor Jan Eric Siemens vom Pharmakologischen Institut der Universität Heidelberg entgegen. Er und sein Team erforschten, wie man mittels Botenstoffen Nervenzellen weniger reizbar machen kann. In der Kategorie Primary Care ging der diesjährige Galenus-Preis an Nalmefen (Selincro ${ }^{\circledR}$ ) von Lundbeck. Hierbei handelt es sich um einen Opioidsystem-Modulator, mit dem sich der Alkoholkonsum bei erwachsenen Patienten mit Alkoholabhängigkeit vermindern lässt. Als gezielte Therapie gegen Knochenmetastasen bei Prostatakrebs erhielt Radium223-dichlorid $\left(\mathrm{Xofigo}^{\oplus}\right)$ von Bayer HealthCare den Preis im Bereich Specialist Care. Seit 2014 werden auch Arzneimittelinnovationen im Bereich Orphan Drugs für Patienten mit seltenen Erkrankungen geehrt. Der diesjährige Preis in dieser Kategorie ging an Ataluren (Translarna ${ }^{\mathrm{TM}}$ ) von PTC Therapeutics. Dabei handelt es sich um die erste zugelassene Therapie mit kausaler Wirkung bei Duchenne-Muskeldystrophie mit zugrunde liegender NonsenseMutation.

Über alle vier Preise entschied wenige Stunden vor der Verleihung eine 14-köpfige Jury um Professor Dr. Erland Erdmann als Vorsitzendem.

\section{CharityAward zum siebten Mal vergeben}

Seit 2009 - und damit zum siebten Mal in Folge - zeichnet Springer Medizin ehrenamtliches Engagement in der Gesundheitsversorgung aus. Von den 49 Bewerbungen schafften es folgende Einrichtungen auf die ersten drei Plätze:
Platz 1 ging an Praxis ohne Grenzen in Hamburg, eine Einrichtung mit 20 Ärzten und weiteren ehrenamtlich Tätigen, die nicht versicherten Menschen dringend benötigte ärztliche Hilfe und Sozialberatung ermöglicht.

Platz 2 belegte das Projekt Babylotse. Die ehrenamtlichen Mitarbeiter der Stiftung des Katholischen Kinderkrankenhauses Wilhelmstift, Hamburg schlieBen Lücken zwischen Krankenhaus und ambulanter ärztlicher Versorgung. Ihre Unterstützung bedürftiger Familien beginnt bereits vor der bevorstehenden $\mathrm{Ge}$ burt eines Kindes und reicht bis zur Hilfe, wenn es beispielsweise um die Bewältigung von Problemen mit chronischen oder schwerstkranken Kindern geht.

Die Landarztmacher aus der Region Arber erreichten schließlich Platz 3. Die Mitglieder dieser Einrichtung engagieren sich für den ärztlichen Nachwuchs, um dem Mangel in ländlichen Gebieten entgegenzuwirken.

Die Bedeutung der von Springer Medizin gestifteten Preise unterstreichen die jeweiligen Schirmherrschaften: Für den Galenus-von-Pergamon-Preis, der in der Branche als inoffizieller Nobelpreis für die Pharmazie gehandelt wird, ist Bundesministerin für Forschung und Bildung Johanna Wanka Schirmherrin. Seit Bestehen des Springer Medizin CharityAward übernimmt der Minister für Gesundheit die Schirmherrschaft.

Uschi Kidane 